against cytotoxic effector cells. Blood. 96:594-600.

9. Medema, J.P., et al. 2001. Blockade of the granzyme B/perforin pathway through overexpression of the serine protease inhibitor PI-9/ SPI-6 constitutes a mechanism for immune escape by tumors. Proc. Natl. Acad. Sci U. S. A. 98:11515-11520.
10. Djerbi, M., et al. 1999. The inhibitor of death receptor signaling, FLICE-inhibitory protein defines a new class of tumor progression factors. J. Exp. Med. 190:1025-1032.

11. Dunn, G.P., et al. 2002. Cancer immunoediting: from immune surveillance to tumor escape. Nat. Immunol. 3:991-998.
12. Hanahan, D., and Weinberg, R.A. 2000. The hall marks of cancer. Cell. 100:57-70.

13. Chouaib, S., Asselin-Paturel, C., Mami-Chouaib, F., Caignard, A., and Blay, J.Y. 1997. The hosttumor immune conflict: from immunosuppression to resistance and destruction. Immunol. Today. 10:493-497.

See the related article beginning on page 617.

\title{
Calcium and the heart: a question of life and death
}

\author{
Andrew R. Marks \\ Center for Molecular Cardiology, Departments of Medicine and Pharmacology, \\ Columbia University College of Physicians and Surgeons, New York, New York, USA \\ J. Clin. Invest. 111:597-600 (2003). doi:10.1172/JCI200318067.
}

The importance of calcium-dependent signaling in the heart has been appreciated for decades. For example, it is well accepted that intracellular calcium release from the sarcoplasmic reticulum (SR) is required for cardiac muscle contraction. Indeed, with each heart beat the calcium concentration in the cytosol of cardiac myocytes is elevated approximately 10 -fold from a resting level of $\sim 100 \mathrm{nM}$ to $\sim 1 \mu \mathrm{M}$.

Presumably, a defect in signaling that prevents effective elevation of cytosolic calcium would impair contractility as the contraction of heart muscle is directly determined by the level of calcium elevation during systole. Similarly, a defect in the removal of calcium from the cytosol during diastole would

\footnotetext{
Address correspondence to: Andrew R. Marks, Center for Molecular Cardiology, Box 65, Columbia University College of Physicians and Surgeons, Room 9-401, 630 West 168th Street, New York, New York 10032, USA. Phone: (212) 305-0270; Fax: (212) 305-3690;

E-mail: arm42@columbia.edu.

Conflict of interest: The author has declared that no conflict of interest exists.

Nonstandard abbreviations used: sarcoplasmic reticulum (SR); nuclear factor of activated T cells (NFAT); protein kinase A (PKA); ryanodine receptor (RyR2); $\mathrm{Ca}^{2+} /$ calmodulin-dependent protein kinase II (CaMKII); cardiac isoform of CaMKII (CaMKII- $\delta$ C).
}

impair cardiac relaxation, which is critically important in that it allows the heart chambers to refill with blood in preparation for the next beat.

\section{Calcium and heart failure}

Indeed, an attractive hypothesis for the mechanism underlying cardiac muscle dysfunction during heart failure, the leading cause of mortality in the developed world, is that impaired calcium release causes decreased muscle contraction (systolic dysfunction) and defective calcium removal hampers relaxation (diastolic dysfunction). Given that the measurement of cellular calcium is relatively straightforward, the obvious experiment required to address this important issue is to measure calcium in heart muscle cells from failing hearts. Such measurements have been done in isolated cardiomyocytes and, though there is a fair amount of variability in the published reports, the data tend to support the concept of a decrease in SR calcium release and a defect in the termination of release. These results imply that there are presumably defects in SR calcium release in vivo. However, there are no data showing that calcium levels are chronically elevated in heart muscle in failing hearts. Such studies await the development of reliable techniques using calcium indicators with adequate signal to noise ratios and detection systems that will permit measurements of intracellular calcium in the living heart in intact organisms.

\section{Calcium and cardiac hypertrophy}

Another disease in which perturbations of calcium signaling have been alluded to is cardiac hypertrophy. Indeed, calcium elevation has been proposed as the trigger for cardiac hypertrophic signaling via the calcium-activated phosphatase calcineurin. Much attention has been focused on this possibility as a potential therapeutic target. Indeed, the initial studies identifying a role for calcineurin in hypertrophic signaling in the heart represented a tour de force, combining beautifully designed in vitro and in vivo studies that clearly demonstrated a physiologically important signaling system (1). An intriguing question is whether or not there are any clinical conditions in which one would actually want to treat (i.e. prevent) cardiac hypertrophy. Indeed, outside the cardiology community, cardiac hypertrophy is often lumped together with heart failure as though they are synonymous. This approach seems to add some excitement to the quest for a cure for cardiac hypertrophy by linking it to heart failure which, as mentioned above, is a leading cause of mortality (over 500,000 deaths per year in the US alone). Cardiac hypertrophy, on the other hand, rarely kills anybody and when it does, death is usually due to cardiac arrhythmias, not the hypertrophy per se. Indeed, most deaths linked to cardiac hypertrophy occur in individuals with inherited forms of the disease most often associated with mutations in one of the contractile proteins. These individuals exhibit abnormal pathology, including disorder in the usually well-ordered arrays 


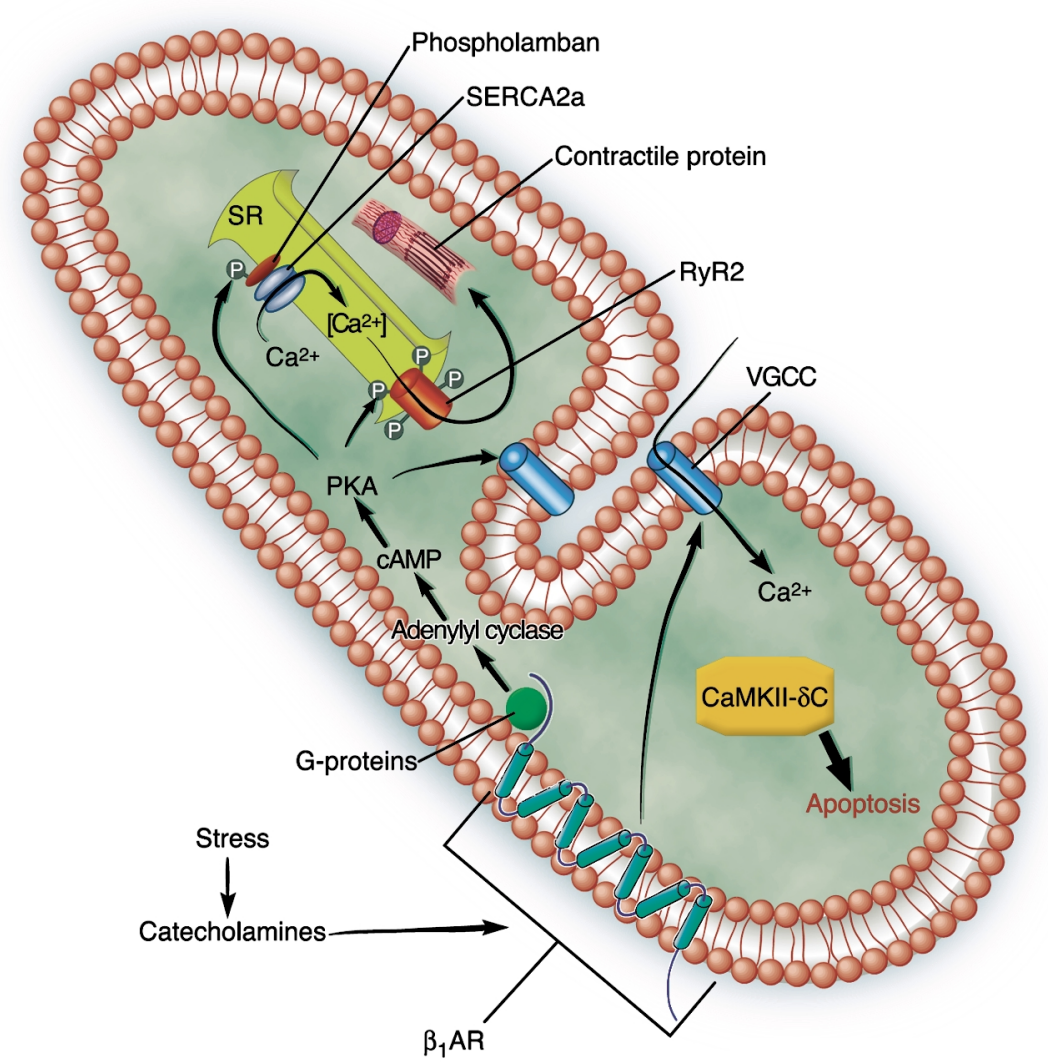

by Ken Beauchamp J. Clin. Invest.

\section{Figure 1}

The $\beta_{1}$-adrenergic receptor/calcium connection. $\beta_{1}$-adrenergic receptor $\left(\beta_{1} A R\right)$ stimulation is known to activate cardiac excitation-contraction (EC) coupling via PKA phosphorylation of the following: (I) the trigger for cardiac EC coupling, the voltage-gated calcium channel (VGCC); (II) the SR calcium release channel RyR2; and (III) the calcium uptake pathway (via PKA phosphorylation of phospholamban, which reduces inhibition of the calcium ATPase SERCA2a). Activation of these proteins by PKA phosphorylation increases systolic SR calcium release which causes both increased cardiac contraction and output. This system results in a maladaptive chronic hyperadrenergic state in heart failure because the damaged heart cannot respond adequately to the adrenergic stimulation and the sympathetic nervous system remains in a futile activated state. This results in PKA-hyperphosphorylated RyR2 channels that can cause a diastolic SR calcium leak (4), which, along with reduced SERCA2a-dependent SR calcium uptake, depletes SR calcium and contributes to contractile dysfunction. Zhou et al. (5) have now added an additional component to this model based on observations that chronic $\beta_{1}$ AR stimulation can activate CaMKII (possibly via increased calcium influx through the VGCC) and cause apoptosis.

of cardiac muscle fibers, suggesting that there may be a substrate for electrical instability. However, the molecular mechanisms underlying the hypertrophy and the arrhythmias in both the inherited and acquired forms of cardiac hypertrophy remain to be elucidated. Moreover, it is quite clear that under certain circumstances some forms of cardiac hypertrophy are an entirely normal physiological response, such as that seen in well-trained athletes or during pregnancy. Other forms of hypertrophy are pathologic in the sense that they ultimately deteriorate into heart failure, but at their initiation cium to activate calcium-dependent enzymes. A good example is that of antigen-dependent $T$ cell activation mediated via the $T$ cell receptor, which results in intracellular calcium release through the inositol 1,4,5-trisphosphate receptor. This calcium release from the ER elevates calcium from $\sim 100 \mathrm{nM}$ to $\sim 1 \mu \mathrm{M}$ resulting in the activation of calcineurin, which subsequently dephosphorylates the nuclear factor of activated $T$ cells (NFAT). Subsequent passage of NFAT into the nucleus activates IL-2 transcription that is required for expansion of the $T$ cell population to fight off infection. There is no solid evidence that small elevations of calcium in the sub- 100 $\mathrm{nM}$ range are sufficient to activate any of the known calcium-dependent enzymes. Therefore, it is very difficult to understand how a calcium-activated enzyme can play a key regulatory (i.e. on/off) role in heart muscle where global cellular calcium is obligated to rise 10 -fold with every heart beat. In most of us, that means a 10 -fold rise of calcium every second or so, and in mice that would equate to one such elevation every $100 \mathrm{~ms}$ given that the mouse heart beats at a rate about 10 times higher than that of humans.

Despite these powerful arguments against a regulatory role for calcium in anything other than contraction in the heart, there remains considerable interest in pursuing the possibility that calcium-dependent signaling pathways are indeed controlling pathologic conditions. Perhaps this interest stems from earlier, unproven, claims that calcium is elevated in heart failure and cardiac hypertrophy. Although, as discussed earlier, no such elevation has ever been documented, the calcium elevation theory regarding heart disease remains in the public eye and offers an attractive therapeutic target - if elevated calcium is causing disease, we'll just have to lower it! How one would go about doing so in heart muscle without disturbing other important functions, such as contraction of the heart muscle, remains to be explained.

Concomitant with the calcium theory of heart disease has been the apoptosis theory of heart failure. The two are linked because it has been proposed that the calcium problem contributes to the apoptosis problem. The apoptosis theory of heart failure is elegantly simple. Something bad hap- 
pens to the heart and triggers programmed death pathways that result in a loss of cardiomyoyctes in sufficient numbers to cause failure of the heart muscle to contract adequately. While there is ample evidence that apoptosis can contribute to cardiac dysfunction in various animal models of heart failure, it remains to be proven that this process plays an important role in causing human heart failure.

\section{Hyperadrenergic state of heart failure}

While these more recent models for heart failure remain to be sorted out, there is a much older story that has been lurking in the background of our understanding of heart failure for over 40 years. This is the story about how hyperadrenergic signaling drives the progressive downhill course of heart failure (2). It has been known for over 40 years that heart failure patients live in a continuous hyperadrenergic state with high levels of circulating catecholamines (3). Many studies have shown that one consequence of this chronic hyperadrenergic state is the downregulation and desensitization of the $\beta_{1}$-adrenergic receptors in the heart. The data showing this effect are strong and have compelled investigators for many years to assume that this meant that the cardiac muscle cell was living in a state of reduced $\beta_{1}$-adrenergic receptor-mediated stimulation. Since the $\beta_{1}$-adrenergic receptors are linked downstream to activation of cAMPdependent protein kinase A (PKA), it was assumed that the targets of this kinase would be under-phosphorylated in failing hearts (Figure 1). Recent studies have shown that this is not the case. Indeed, it turns out that the major SR calcium release channel in the heart, the ryanodine receptor (RyR2), is PKA hyperphosphorylated in failure hearts. This results in leaky channels that may help explain the reduced calcium signal observed in heart failure (possibly due to depletion of SR calcium pools via the leaky channel) (4).

\section{Linking $\beta_{1}$-adrenergic signaling to CaMKII}

In this issue of the JCI, Zhu et al. (5) now provide a novel and intriguing possibility that could explain additional adverse effects of the chronic hyper- adrenergic state of heart failure. Moreover, their surprising findings implicate that $\beta_{1}$-adrenergic receptors mediate signaling not via PKA, as would be expected, but through another kinase not previously thought to be downstream of $\beta_{1}$-adrenergic receptors, the $\mathrm{Ca}^{2+} /$ calmodulin-dependent protein kinase (CaMKII). What is exciting about this study is that it links CaMKII to $\beta_{1}$-adrenergic receptors for the first time. Whether or not the activation of apoptotic pathways demonstrated in vitro by Zhu et al. turns out to be an important contributor to heart failure in humans, this initial description of a novel and unexpected connection between two signaling pathways is a significant addition to the continuously expanding repertoire of crosstalk between signals in biological systems. This finding represents yet another example of why purely pharmacologic-based studies need to be viewed with skepticism, especially when claims are made about drug specificity. Indeed, even if the drugs are specific, unrecognized cross-talk downstream of the receptor may confound interpretation of the data. In the present case, despite $\beta_{1}$-adrenergic receptor activation, the downstream signaling via CaMKII is not inhibited by PKA inhibitors! Indeed, this is not the first example of non-PKA signaling downstream of $\beta_{1}$-adrenergic receptors. Elegant studies by Lefkowitz and colleagues have shown that $\beta_{2}$-adrenergic receptor complexes can participate in nonreceptor protein tyrosine kinase signaling (6).

The Zhu et al. (5) study proposes that chronic stimulation of $\beta_{1}$-adrenergic receptors (this would mimic one aspect of the chronic hyperadrenergic state that afflicts heart failure patients) results in cardiomyocyte cell death due to activation of CaMKII signaling via up regulation of calcium. They provide support for this hypothesis using a variety of in vitro manipulations including pharmacologic studies where CaMKII inhibitors, but not PKA inhibitors, were shown to block the induction of apoptosis by isoproterenol, and overexpression of the cardiac isoform of CaMKII (CaMKII- $\delta C$ ) was demonstrated to enhance the pro-apoptotic effect of isoproterenol.

It should be clear from the first part of this commentary that an important unexplained question is how would CaMKII activity be increased in vivo? Put another way, how does CaMKII distinguish between the obligatory 10 -fold elevations of calcium required for heart muscle contraction and the pathologic signals elicited by the chronic hyperadrenergic state? In this sense, the CaMKII story proposed by Zhu et al. (5) shares a common feature with the calcineurin story alluded to earlier (1). Both suggest that pathologic processes - cell death in the first case and cellular hypertrophy in the second - are triggered by the same signal, increased cellular calcium. It is entirely possible that both stories are correct in identifying novel signaling pathways in the heart but that neither actually requires perturbations of calcium signaling as the explanation for how these signals are initiated. In the case of calcineurin, an alternative explanation for the link between an increase in calcineurin activity and hypertrophic signaling is that the levels of calcineurin are elevated as part of growth factor-stimulated remodeling of the heart. There may also be a reduction in the activity of a kinase(s) paired with calcineurin. Both of these could occur in a calcium-independent manner.

Similarly, an alternative hypothesis to that of Zhu et al. (5) is that CaMKIIdependent apoptotic signals are indeed mediated via $\beta_{1}$-adrenergic receptors but they do not involve calcium. For example, increased CaMKII-mediated effects could reflect a downregulation of the protein phosphatase that is paired with CaMKII, or an increase in CaMKII protein levels due to transcriptional effects. If one accepts that it would be incompatible with life to have a sustained elevation of calcium in the heart at levels sufficient to activate CaMKII (the result would be a "stone heart" or rigor mortis), then another possibility is that localized signaling complexes could be exposed to chronic elevations of calcium (e.g. through a "leaky" channel) that would not be reflected in global elevations of calcium. Improved imaging techniques are now capable of measuring such microdomains of calcium (7). However, such studies will be limited to ex vivo measurements in isolated cardiomyocytes. Another important task is to identify the role of $\beta_{1}$-adrenergic receptor-mediated activation of CaMKII in normal physiology. 
Notwithstanding these important unresolved questions, the Zhu et al. (5) study should open up a new area of investigation as additional studies probe the connections between $\beta_{1}$-adrenergic receptor-mediated signaling and CaMKII-dependent effects. If future studies support the proposal by Zhu et al., that one of the consequences of prolonged $\beta_{1}$-adrenergic receptor stimulation is cardiomyocyte cell death via a CaMKII-mediated pathway, it will indeed be an important contribution to our understanding of the pathogenesis of heart failure that will suggest novel therapeutic targets.

1. Molkentin, J.D., et al. 1998. A calcineurindependent transcriptional pathway for cardiac hypertrophy. Cell. 93:215-228.

2. Marks, A.R., Reiken, S., and Marx, S.O. 2002. Progression of heart failure: is protein kinase a hyperphosphorylation of the ryanodine receptor a contributing factor? Circulation. 105:272-275.

3. Chidsey, C.A., Harrison, D.C., and Braunwald, E. 1962. Augmentation of plasma norepinephrine response to exercise in patients with congestive heart failure. N. Engl. J. Med. 267:650.

4. Marx, S.O., et al. 2000. PKA phosphorylation dissociates FKBP12.6 from the calcium release channel (ryanodine receptor): defective regulation in failing hearts. Cell. 101:365-376.

5. Zhu, W.-Z., et al. 2003. Linkage of $\beta_{1}$-adrenergic stimulation to apoptotic heart cell death through protein kinase $\mathrm{A}$-independent activation of $\mathrm{Ca}^{2+} /$ calmodulin kinase II. J. Clin. Invest. 111:617-625. doi:10.1172/JCI200316326.

6. Luttrell, L.M., et al. 1999. Beta-arrestin-dependent formation of beta2 adrenergic receptor-Src protein kinase complexes. Science. 283:655-661.

7. Zaccolo, M., Magalhaes, P., and Pozzan, T. 2002 Compartmentalisation of cAMP and $\mathrm{Ca}(2+)$ signals. Curr. Opin. Cell. Biol. 14:160-166.

\section{Soluble VEGF receptor Flt1: the elusive preeclampsia factor discovered?}

\section{Aernout Luttun and Peter Carmeliet}

The Center for Transgene Technology and Gene Therapy, Flanders Interuniversitary Institute for Biotechnology, Katholieke Universiteit Leuven, Leuven, Belgium

J. Clin. Invest. 111:600-602 (2003). doi:10.1172/JCI200318015.

The occurrence of seizures (eclampsia, from the Greek "eklampsis," sudden flashing) has been a long-known and feared complication of pregnancy, often killing both mother and child. Preeclampsia, or the condition preceding full-blown eclampsia, affects up to $5 \%$ of pregnant women and is diagnosed by the onset of hypertension and proteinuria in the second trimester (1). Preeclampsia may eventually progress to glomerular malfunction, thrombocytopenia, liver and brain edema, and associated lifethreatening seizures (2) (Figure 1). Preeclampsia has been sometimes

\footnotetext{
Address correspondence to: Peter Carmeliet, Center for Transgene Technology and Gene Therapy, Flanders Interuniversitary Institute for Biotechnology, Katholieke Universiteit Leuven, Campus Gasthuisberg, Herestraat 49, B-3000, Leuven, Belgium.

Phone: 32-16-34-57-72; Fax: 32-16-34-59-90; E-mail: peter.carmeliet@med.kuleuven.ac.be. Conflict of interest: The authors have declared that no conflict of interest exists. Nonstandard abbreviations used: placental growth factor (PlGF); fms-like tyrosine kinase 1 (Flt1); soluble Flt1 (sFlt1).
}

termed the "disease of theories," as several models for its pathogenesis have been proposed. But, as of today, no satisfactory unifying hypothesis has emerged (1). The restricted occurrence of preeclampsia to humans and primates and the lack of a suitable animal model have hampered the understanding of its pathogenesis (3). In this issue of the JCI, S.E. Maynard et al. (4) report the novel insight that circulating levels of two angiogenic growth factors, VEGF and placental growth factor (PlGF), may play a more important role than previously believed. In particular, the authors propose that, in pregnant women with preeclampsia, the placenta produces elevated levels of the soluble fms-like tyrosine kinase 1 (sFlt 1 ) receptor, which captures free VEGF and PIGF. As a result, the normal vasculature in the kidney, brain, lungs, and other organs is deprived of essential survival and maintenance signals and becomes dysfunctional (Figure 1). As the authors show in their rodent model, this may lead to the development of hypertension and renal dis- ease, reminiscent of preeclampsia in humans. In another study in this issue, V. Eremina et al. (5) provide additional evidence for a critical role of VEGF in renal disease during preeclampsia. These authors demonstrate that mice lacking one VEGF allele in renal podocytes develop the typical renal pathology found in pregnant women with preeclampsia. These studies therefore shed unprecedented light on the pathogenesis of preeclampsia and offer novel therapeutic opportunities for this disease.

\section{sFlt1: a likely candidate preeclampsia factor}

For the fetus to develop normally, it must receive sufficient oxygen and nutrients (6). These are supplied via the maternal spiral arteries in the uterus. During normal pregnancy, cytotrophoblasts convert from an epithelial to an endothelial phenotype (a process termed pseudo-vasculogenesis) and invade maternal spiral arteries. This vascular remodeling increases the bulk flow and the supply of nutrients and oxygen to the fetus by the end of the first trimester $(7,8)$ (Figure 1). Vascular factors such as VEGF, angiopoietins, and ephrins have been implicated in this process (7). In preeclampsia, pseudo-vasculogenesis is defective, and the resultant placental ischemia has been proposed to trigger the release of unknown placenta-derived factors. The latter would induce systemic endothelial dysfunction and thereby contribute to the renal, cardiovascular, and neurological defects of preeclampsia (Figure 1). Despite intensive efforts, the precise nature of the placenta-derived 